\title{
Eudragit E100 and Polysaccharide Polymer Blends as Matrices for Modified-Release Drug Delivery I: Physicomechanical Properties
}

\author{
Ndidi C Ngwuluka*, Elijah I Nep, Nelson A Ochekpe, Patricia O Odumosu and \\ Patrick O Olorunfemi \\ Biomaterials and Drug Delivery Unit, Faculty of Pharmaceutical Sciences, University of Jos, 930001, Nigeria
}

*For correspondence: Email: Ndidi.Ngwuluka@biodrudel.com; Tel: +234-73-290269

Received: 29 October 2015

Revised accepted: 7 October 2015

\begin{abstract}
Purpose: To compare the effects of two states of polymer/polymer blending (dry and aqueous/lyophilized) on the physicomechanical properties of tablets, containing blends of locust bean gum (LB) with Eudragit@ E100 (E100) and sodium carboxymethylcellulose (SCMC) as matrices.

Methods: LB, SCMC and E100 were blended in their dry (as purchased) state or modified by aqueous blending and subsequent lyophilization, prior to use as matrices in tablets. The polymer blends were characterized by infra-red spectroscopy (FTIR), flow and compressibility tests, as well as physicomechanical analysis of their tablets.

Results: No significant variations were noticeable in the FTIR peaks of the individual polymers in the dry and the aqueous/lyophilized states. Aqueous/lyophilized blending of the polymers resulted in better flow properties. The aqueous/lyophilized matrices were denser with improved mechanical strength and the tablets were harder than those produced from dry blended polymers.

Conclusion: Dry blending of LB with E100 and SCMC greatly improved the physicomechanical properties of the tablets. This was further enhanced by aqueous/lyophilized blending.
\end{abstract}

Keywords: Drug delivery, Polymer blend, Eudragit, Locust bean gum, Levodopa, Sodium carboxymethylcellulose, Matrix, Physicomechanical properties

Tropical Journal of Pharmaceutical Research is indexed by Science Citation Index (SciSearch), Scopus, International Pharmaceutical Abstract, Chemical Abstracts, Embase, Index Copernicus, EBSCO, African Index Medicus, JournalSeek, Journal Citation Reports/Science Edition, Directory of Open Access Journals (DOAJ), African Journal Online, Bioline International, Open-J-Gate and Pharmacy Abstracts

\section{INTRODUCTION}

Polymers may be used to retard the rate of release of drugs with the aim of producing a relatively uniform release over time. When polymers utilized are not able to effectively control drug release to a uniformed rate, the polymers may be modified to achieve this purpose. Some methods of modification of polymers include: derivative formation [1], grafting [2], crosslinking [3] and polymer-polymer blending [4].
Polymer-polymer blending provides a convenient alternative to the synthesis of new polymers. The blending of two or more polymers may generate a new polymer with enhanced and possibly desired drug delivery properties in comparison to the individual polymers. Furthermore, physical modification, such as aqueous mixing and drying, may vary the physicochemical behaviour (strength and deformability, for instance) of a polymer without altering its chemical structure or molecular distribution [5]. 
Locust bean gum (LB) is a galactomannan extracted from the seeds of the carob tree. It is a non-ionic polysaccharide with a $\mathrm{pH}$ independent hydration rate. When used in matrix tablets, locust bean gum has been shown to undergo rapid erosion of the hydration layer, resulting in a burst release which was seen to be absent when the gum was used in combination with xanthan, a hydrophilic anionic heteropolysaccharide $[6,7]$.

In the present study, locust bean gum was blended with sodium carboxymethylcellulose (SCMC) and Eudragit® E100 (E100), and the effect of dry and aqueous blending on the physicomechanical properties of the polymer was evaluated. E100 is a cationic copolymer of dimethylaminoethyl methacrylate, butyl methacrylate, and methyl methacrylate while SCMC is a semi-synthetic anionic polysaccharide. If the two states of blending significantly affect the physicomechanical properties of the tablets, it will be expected that the release of active pharmaceutical ingredients from the tablets can also be modulated.

\section{EXPERIMENTAL}

\section{Materials}

Eudragit® E100 (Evonik Röhm GmbH \& Co. KG, Darmstadt, Germany), sodium carboxymethylcellulose (SCMC, Fluka Biochemika, Medium viscosity, Sigma-Aldrich Chemie $\mathrm{GmbH}$, Buchs, Switzerland), locust bean from Ceratonia siliqua seeds (Sigma-Aldrich Inc, Steinheim, Germany), acetic acid glacial, hydrochloric acid (Rochelle Chemicals, Gauteng, South Africa), silica (Saarchem, Krugersdorp, South Africa), magnesium stearate (Merck Chemicals (PTY) LTD, Gauteng, South Africa), barium sulphate, pullulan from Aureobasidium pullulans, 3-(3,4Dihydroxyphenyl)-L-alanine (levodopa, SigmaAldrich Inc., Steinheim, Germany).

\section{Blending of polymers}

Sodium carboxymethylcellulose (SCMC), Eudragit® (E100) and locust bean (LB) were used in their dry (as purchased) and modified (aqueous blending and subsequent lyophilization) states. They were used in formulation of tablet matrices individually as well as in combination (the three polymers combined). For the modified states of the individual polymers, the following procedures were undertaken. SCMC (4.2 g) was dissolved in $50 \mathrm{~mL}$ of water and thereafter lyophilized. LB (4.2 g) was dispersed in $50 \mathrm{~mL}$ of water and thereafter lyophilized. E100 (4.2 g) was dissolved in $50 \mathrm{~mL}$ of $0.1 \mathrm{~N}$ acetic acid and then lyophilized. The dry blend of the three polymers was obtained by mixing the three polymers, E100, SCMC and LB uniformly. Furthermore, the modified state of the tripolymer was prepared. To prepare modified state of the tripolymer; E100 and SCMC were dissolved separately as described above. E100 was then added into SCMC and allowed to interact under vigorous agitation for 2-3 h. After ascertaining no further visible change was observed in the medium, LB was added and agitated for $15 \mathrm{~min}$ and the blend was thereafter lyophilized. The three polymers (E100:LB:SCMC) were utilized in the ratios $1: 1: 0.5 ; 1: 0.5: 1$ and $1: 1: 1$ in both dry and modified states. After lyophilization, the polymers/polymer blends were milled for spectroscopy, flow properties analysis and subsequent formulation by direct compression.

\section{Fourier transform infra-red (FTIR) spectroscopy}

Fourier Transform Infra-Red spectra were obtained with a PerkinElmer spectrometer (PerkinElmer Spectrum 100, Beaconsfield, United Kingdom) over a range of $4000-650 \mathrm{~cm}^{-1}$ for each polymer/polymer blend to assess any structural difference. PerkinElmer Spectrum 100 utilizes Universal Attenuated Total Reflectance (UATR) for speedy analysis and reproducibility. The samples were separately placed in direct contact with the ATR crystal and the pressure arm positioned directly on the sample and using the instrument software, the spectra were obtained over the specified range above.

\section{Assessment of flow and compressibility properties}

Techniques such as flow rate, angle of repose, bulk density, tapped density and compressibility index were undertaken to compare the flow and compressibility properties of polymer/polymer blend in its dry and modified states applying the equations as stated by Ngwuluka et al [8]. Each polymer/polymer blend was blended with levodopa, barium sulfate, pullulan, magnesium stearate and silica. Thereafter, $5 \mathrm{~g}$ of each blend was used for the techniques stated above.

\section{Direct compression of tablets}

To prepare tablet matrices, the model drug, levodopa (100 mg), a high density salt - barium sulphate (234 mg), adhesive (Pullulan - $100 \mathrm{mg}$ ), lubricant (Magnesium stearate $-10 \mathrm{mg}$ ) and glidant (silica - $55 \mathrm{mg}$ ) were blended with each polymer/polymer blend $(500 \mathrm{mg})$ in their dry and modified states. Each blended batch was 
compressed using a hydraulic tablet press (Carver Industries Inc., Wabash, IN, USA) at $29.4 \mathrm{KN}$. The excipients were chosen to formulate tablet matrices for narrow absorption window drugs and levodopa was chosen as the model drug. Hence, for a polymer or its blend, there were two batches; one batch using the polymer/polymer blend in its dry state and another using the polymer/polymer blend in its modified state.

\section{Physicomechanical analysis}

To compare the physicomechanical strength of the tablet matrices, force-distance profiles of the matrices were obtained using a texture analyzer (TA.XTplus, Stable Microsystems, Surrey, UK). A $2 \mathrm{~mm}$ flat-tipped steel probe was used to perform the matrix hardness and deformation energy while matrix resilience was carried out with a 36 $\mathrm{mm}$ cylindrical probe fitted to the texture analyzer and the data captured through texture exponent software (Version 3.2). The parameter settings employed were $1 \mathrm{~mm} / \mathrm{sec}$ for pre-test speed, 0.5 $\mathrm{mm} / \mathrm{sec}$ for test speed, $1 \mathrm{~mm} / \mathrm{s}$ for post-test speed, $40 \mathrm{~N}$ for compression force (employed for matrix hardness and deformation energy), $25 \%$ for compression strain (employed for matrix resilience) and $0.5 \mathrm{~N}$ for trigger force.

\section{Assessment of densities of the tablet polymer matrix}

Densities of the tablet matrices were determined by obtaining the diameters and the thicknesses (to calculate volumes) of the tablets, using 0-150 $\mathrm{mm}$ electronic digital caliper (Hangzhou United Bridge Tools Co Ltd, Zhejiang, China) and gravimetric determination of weights.

\section{Statistical analysis}

Basic statistics, such as standard deviation was used to analyze the physicochemical data such as those of flow rate, angle of repose, hardness, densities and resilience.

\section{RESULTS}

As a solution of E100 is added to SCMC, a color change was observed. Two transparent polyelectrolytic solutions interact under agitation to produce white strands running through SCMC gel. As agitation continued, the E100-SCMC blend appeared entangled with increased viscosity. These polymers interacted at different degrees based on the ratio of each polymer in the blends. The agitation was undertaken for $3 \mathrm{~h}$ before locust bean (LB) was added which further increased the viscosity. After lyophilization, polymer/polymer blend appeared like a porous wafer, taking the shape of the petri dish used (or the frozen polymer before sublimation and drying). Subsequently, milling generated a more voluminous powder than the same weight of the native polymer.

\section{Fourier transform infra-red (FTIR) spectra}

The FTIR spectra of lyophilized and dry locust bean gum are shown in Figure 1a. Not much difference is observed between the spectrum of lyophilized (dispersed in water and lyophilized) LB gum and that of dry LB gum except at 1537.9 $\mathrm{cm}^{-1}$. While dry polymer (utilized as purchased) had a peak at $1537.9 \mathrm{~cm}^{-1}$, lyophilized did not. E100's characteristic peak can be seen at $1722.39 \mathrm{~cm}^{-1}$ as shown in Figure 1b. It was also observed in F1, F2 and F3 (Figures 1c). However, the intensity of absorbance peak was significantly reduced in the polymer blends and there was no shift of the peak. The peak falls within the carbonyl group region - 1710 to 1740 $\mathrm{cm}^{-1}$. The broad peaks found in SCMC and LB between 3600 and $3000 \mathrm{~cm}^{-1}$ are indicative of the $\mathrm{OH}$ stretches of galacturonic units. However, the intensity of absorbance at this region reduced in the polymer blends.

\section{Flow and compressibility properties}

From Table 1, it will be observed that lyophilization increased the angles of repose of the polymers and their blends. It also decreased their flow rate with the exception of LB. The dry polymers and polymer blends exhibited low angles of repose $(\leq 10]$.

\section{Physicomechanical characteristics}

Table 2 shows the comparative analyses of the mechanical strength of dry and modified state polymers/polymer blends. A classic example of the modification of physicomechanical strength proffered by aqueous blending and subsequent lyophilization was observed with LB. After direct compression, tablets prepared with LB polymer were visibly friable without much pressure which implied that the tablets may crumble into particles on storage and handling. Modified state LB tablets were harder, the resilience of LB to stress was improved and the tablets were less deformed than those of dry state (native) LB. The native polymers (as purchased) and their blends in the dry state absorbed more of the energy (stress applied) and were less resilient than the aqueous blended and lyophilized polymer blends. 

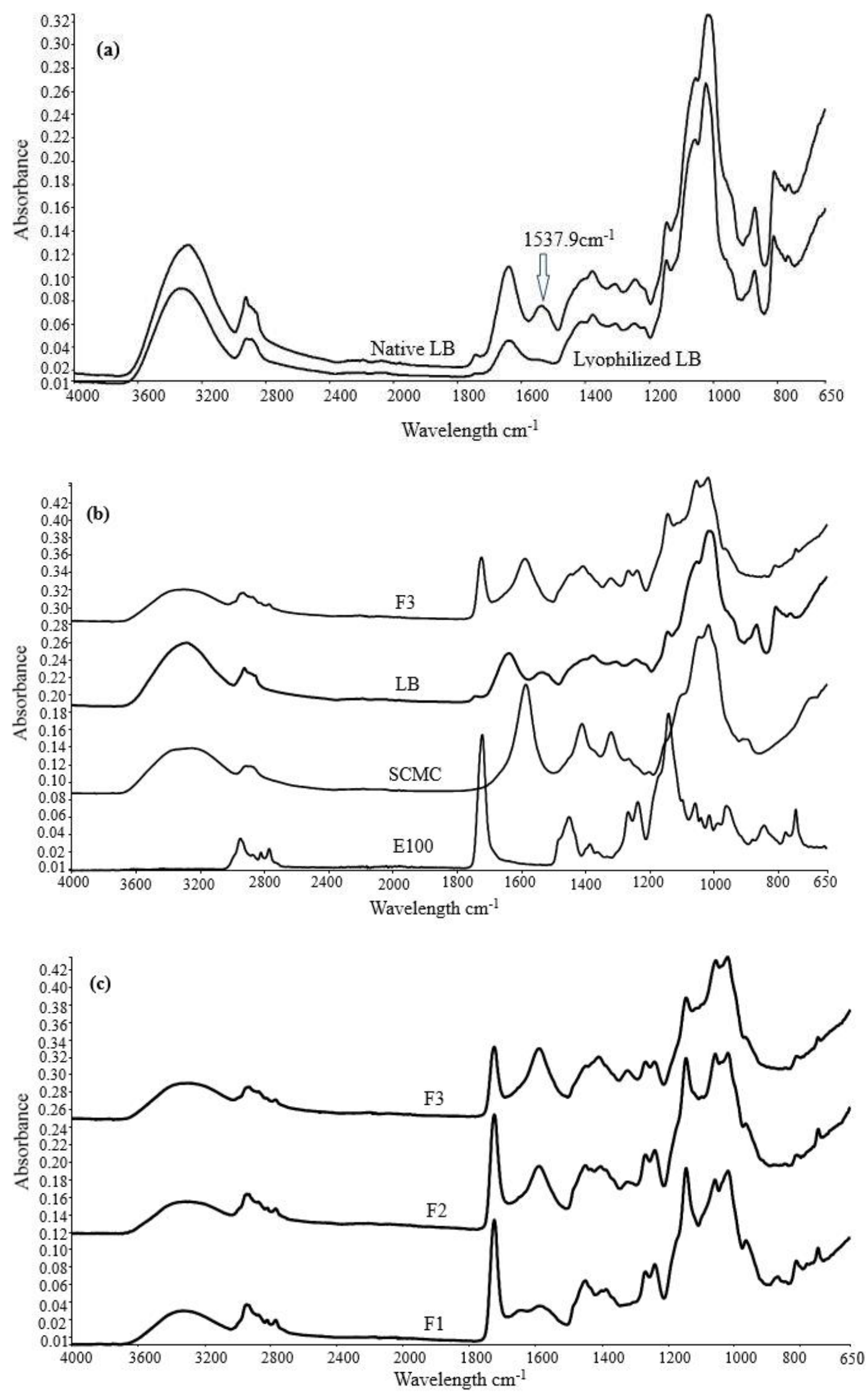

Figure 1: Fourier Transform Infra-Red (FTIR) spectra of: (a) Native and lyophilized locust bean, (b) polymer blend F3 and the native polymers - E100, SCMC and LB and (c) polymer blends F1, F2 and F3 
Table 1: Flow properties of polymer/polymer blends in dry and lyophilized states

\begin{tabular}{lcccc}
\hline $\begin{array}{l}\text { Formulation } \\
\text { code }\end{array}$ & Flow rate $(\mathbf{m m} / \mathbf{s})$ & $\begin{array}{c}\text { Angle of repose } \\
\left({ }^{\circ}\right)\end{array}$ & $\begin{array}{c}\text { Compressibility } \\
(\%)\end{array}$ & $\begin{array}{c}\text { Hausner's } \\
\text { Quotient }\end{array}$ \\
\hline SCMCaq & $22.14 \pm 0.61$ & $32.20 \pm 0.71$ & 34.38 & 1.52 \\
SCMCp & $39.53 \pm 0.44$ & $6.28 \pm 0.71$ & 39.13 & 1.64 \\
E100aq & $13.74 \pm 1.27$ & $30.54 \pm 0.00$ & 30.77 & 1.44 \\
E100p & $77.98 \pm 0.08$ & $10.20 \pm 2.12$ & 31.11 & 1.45 \\
LBaq & $16.18 \pm 1.46$ & $31.80 \pm 2.12$ & 38.24 & 1.62 \\
LBp & $16.16 \pm 3.58$ & $13.50 \pm 4.24$ & 35.71 & 1.56 \\
F1aq $(1: 1: 0.5)$ & $14.31 \pm 0.00$ & $30.96 \pm 0.00$ & 36.36 & 1.57 \\
F1p $(1: 1: 0.5)$ & $117.24 \pm 0.15$ & $6.84 \pm 0.71$ & 35.56 & 1.55 \\
F2aq $(1: 0.5: 1)$ & $25.26 \pm 0.80$ & $29.25 \pm 0.71$ & 29.17 & 1.41 \\
F2p $(1: 0.5: 1)$ & $115.65 \pm 0.15$ & $6.84 \pm 0.71$ & 34.09 & 1.52 \\
F3aq $(1: 1: 1)$ & $26.60 \pm 0.09$ & $26.10 \pm 1.41$ & 32.35 & 1.47 \\
F3p $(1: 1: 1)$ & $149.12 \pm 0.01$ & $5.71 \pm 0.00$ & 30.95 & 1.45 \\
\hline 1 aq signifies polymer/polymer blend in aqueous blending and lyophilized state; ${ }^{2} p$ signifies polymer/polymer in \\
dry blending. F1; F2 and F3 are polymer blends of SCMC, E100 and LB
\end{tabular}

Table 2: Physicomechanical data of tablet matrices of polymers and their blends

\begin{tabular}{lccc}
\hline $\begin{array}{c}\text { Formulation } \\
\text { Code }\end{array}$ & $\begin{array}{c}\text { Hardness } \\
(\mathbf{N} / \mathbf{m m})\end{array}$ & $\begin{array}{c}\text { Absorption Energy } \mathbf{x} \\
\mathbf{1 0 ^ { - 2 }} \mathbf{( N m )}\end{array}$ & \% Resilience \\
\hline SCMCaq & $35.10 \pm 0.07$ & $1.3 \pm 7.07 \times 10^{-4}$ & $46.49 \pm 0.79$ \\
SCMCp & $35.73 \pm 0.06$ & $1.3 \pm 7.07 \times 10^{-4}$ & $42.09 \pm 0.17$ \\
E100aq & $38.18 \pm 0.08$ & $1.2 \pm 7.07 \times 10^{-4}$ & $47.32 \pm 0.42$ \\
E100p & $6.20 \pm 0.06$ & $3.1 \pm 1.4 \times 10^{-3}$ & $43.78 \pm 0.77$ \\
LBaq & $34.73 \pm 0.18$ & $1.2 \pm 7.07 \times 10^{-4}$ & $46.95 \pm 0.94$ \\
LBp & $7.28 \pm 0.07$ & $3.0 \pm 1.4 \times 10^{-3}$ & $34.51 \pm 0.09$ \\
F1aq $(1: 1: 0.5)$ & $39.26 \pm 0.14$ & $1.2 \pm 7.07 \times 10^{-4}$ & $45.39 \pm 0.70$ \\
F1p $(1: 1: 0.5)$ & $29.88 \pm 0.04$ & $1.7 \pm 7.07 \times 10^{-4}$ & $42.33 \pm 0.81$ \\
F2aq $(1: 0.5: 1)$ & $38.35 \pm 0.09$ & $1.4 \pm 7.07 \times 10^{-4}$ & $44.25 \pm 0.45$ \\
F2p $(1: 0.5: 1)$ & $33.73 \pm 0.06$ & $1.4 \pm 7.07 \times 10^{-4}$ & $41.85 \pm 0.97$ \\
F3aq $(1: 1: 1)$ & $38.84 \pm 0.08$ & $1.2 \pm 7.07 \times 10^{-4}$ & $46.23 \pm 0.74$ \\
F3p $(1: 1: 1)$ & $30.34 \pm 0.06$ & $1.6 \pm 7.07 \times 10^{-4}$ & $41.45 \pm 0.45$ \\
\hline
\end{tabular}

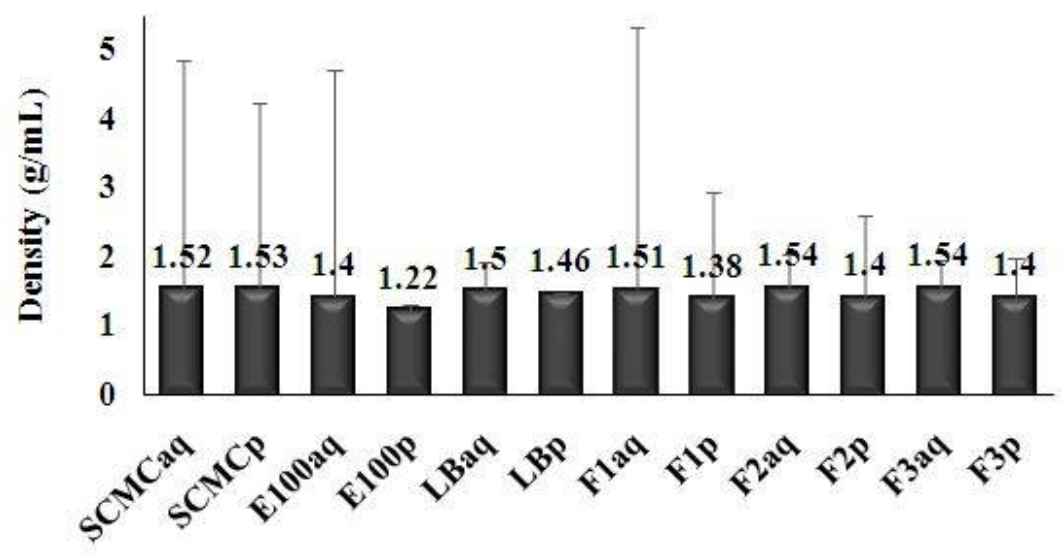

\section{Formulations}

Figure 2: Bar chart depicting the densities of the formulations of the native polymers and their blends

Density of tablet matrices of polymer and their blends

All the formulations (Figure 2) fell within the recommended density range (1.3 to $2.8 \mathrm{~g} / \mathrm{mL}$ ) for gastroretentive tablets except $\mathrm{E} 100$ in dry state which had a density of $1.22 \mathrm{~g} / \mathrm{mL}$. Generally, the tablet matrices formulated with lyophilized polymers/polymer blends exhibited higher densities than the dry polymers/polymer blends.

\section{DISCUSSION}

$\mathrm{E} 100$ is a cationic polyelectrolyte, while SCMC is an anionic polyelectrolyte. The interaction of these two transparent polyelectrolytic solutions 
under agitation may have produced a polyelectrolyte complex. The increase in viscosity of the E100-SCMC blend upon addition of LB is expected as locust bean is a viscosity enhancing/thickening agent. The powder volume of the lyophilized polymer/polymer blend may be due to the introduction of channels during sublimation such that when the material is milled, it occupies more volume than the native material(s). Furthermore, lyophilization may affect nature of polymers changing them from semi-crystalline to amorphous or a blend of both. The impacts of lyophilization on polymorphism of materials have been reported by other researchers $[9,10]$.

FTIR was undertaken due to the visible colour change observed during aqueous blending. The focus of FTIR spectroscopy in this study was to compare the individual polymers with their blends. However, based on the significant change in LB drug delivery behaviour, the FTIR spectrum of the native was compared with that of the lyophilized. The absence of the peak at $1537.9 \mathrm{~cm}^{-1}$ after lyophilization of LB may be due to self-association of galactomannan chains in aqueous solutions which may have occurred when locust bean was dispersed in water before lyophilization [11-13]. To further postulate; the peak in dry LB may be due to asymmetric stretching of the free carboxyl groups and the disappearance in the lyophilized, may indicate that the lyophilized LB has become more hydrophobic, having a higher degree of esterification. The galactomannan chains may have utilized the free carboxyl groups in hydrophobic self-association through intramolecular and/or intermolecular bondings.

The decrease in the intensity of absorbance of E100's characteristic peak, suggests that the covalent bond of the carbonyl group may have interacted with the polyelectrolyte, SCMC. Broad peaks between 3600 and $3000 \mathrm{~cm}^{-1}$ indicative of the $\mathrm{OH}$ stretches of galacturonic units are expected as LB is a polysaccharide and SCMC is a polysaccharide derivative. The quantities of materials (SCMC and LB) responsible for this broad peak in the polymer blends are less compared with the quantities of the polysaccharide materials alone.

In summary, there appeared to be no significant shift in peaks. The peaks at the group frequency region $\left(4000-1450 \mathrm{~cm}^{-1}\right)$ of the native polymers were observed in the polymer blends, F1, F2 and F3 with reduction in intensity of absorbance. In the fingerprint region $\left(1450-600 \mathrm{~cm}^{-1}\right)$, there appeared to be peaks and super-imposition of peaks and no unique peak different from those of the native polymers. The spectra obtained and the visible colour change during blending suggests that the reaction between the polymers may be a complexation reaction especially between E100 and SCMC, as these are polyelectrolytes. The proportions of polymers in each blend also influenced the intensity of absorbance of peaks. This can be clearly seen in the frequency region between 1700 and 1500 $\mathrm{cm}^{-1}$. This region has a peak characteristic to SCMC and the intensity of absorbance varied in blends F1, F2 and F3. F1 has the least of absorbance which is due to this blend having the least proportion of SCMC. The peak was found in the spectra of the polymer blends at slightly but not significantly different frequencies - F1 at 1587.39, F2 at 1589.72 and $\mathrm{F} 3$ at 1589.85.

The United States Pharmacopeia (USP) describes powders with angle of repose between 25 and $30^{\circ}$ as having excellent flow properties, while compressibility index range of $32-37 \%$ and Hausner's ratio range of $1.46-1.59$ as having very poor flow properties [14]. Furthermore, it clarifies that angle of repose, compressibility index and Hausner's ratio are not intrinsic properties of a powder but depend on the method used. Generally, considering the angle of repose, the polymer blends in lyophilized states exhibited better flow properties $\left(\leq 30^{\circ}\right)$ than the individual polymers in their dry and lyophilized states. The dry polymers and polymer blends exhibited low angles of repose ( $\leq 10)$. This was envisaged as they were not as bulky as the lyophilized polymers and polymer blends, thereby occupying less height and giving rise to low angles of repose $[\theta=\tan -1$ (height/0.5base)]. In addition, the compressibility indices and Hausner's ratios obtained indicated that most of the polymers and their blends exhibited poor flow properties except E100p and E100aq, F2aq and F3p. However, a glidant could be used to improve flow.

Direct compression is one of the techniques used in the compaction of powders into tablets, especially for formulation of thermolabile and moisture-sensitive drugs. Direct compression is increasingly becoming a convenient technique to compact solid dosage forms, due to its cost effectiveness as operations steps are reduced leading to shorter processing time, lower energy consumption, and more production outputs with less excipients. In the past, direct compression could not be used for drugs with poor flowing properties and drug content of about $30 \%$ and also the challenge of segregation was also a limitation $[15,16]$. However, in recent times, an increasing number of drugs can now be directly compressed due to the development and 
production of excipients, specially designed for direct compression [15]. Aqueous blending and lyophilization enhanced the direct compressibility of the polymers as evidenced by LB and E100. The physicomechanical analyses confirmed the visual observations.

Generally, the physicomechanical results obtained for the formulations indicate that blending of polymers, both in dry and aqueous states improved the mechanical strength of the tablet matrices in comparison to those of the individual polymers. Lyophilization is a process with three stages - freezing, ice sublimation and water desorption - and the first stage largely determines the physical properties of the dried material [17]. Mechanical strength or hardness is a physical property which has been enhanced by lyophilization. Matrix resilience is described as the ability of a strained matrix to regain its size and shape after deformation due to stress [18]. The hardness of lyophilized polymers/polymer blends indicates that they will be able to withstand stress, pressures and handling during transportation. Their rigidity may have been enhanced by the intramolecular hydrogen bonding [11] that may have been strengthened in the presence of bound-water before drying. Furthermore, hardness of a tablet is dependent on its ability to undergo plastic deformation [19, 20]. Lyophilization may have improved their plastic deformation thereby increasing the cohesive forces between the polymer particles which led to harder tablets.

The formulation of high density delivery systems is one of the techniques utilized for gastroretention. These systems are expected to sink to the antrum of the stomach, preventing immediate evacuation and as a result, narrow absorption window drugs are retained longer at their sites of absorption. The model drug used in this study, levodopa has its site of absorption at the proximal small intestine.

Hence, the longer the drug delivery system is retained in the stomach, and the drug is released over time from the system, the more available levodopa is to the proximal small intestine. The tablet matrices formulated with lyophilized polymers/polymer blends exhibited higher densities than the dry polymers/polymer blends possibly due to enhancement of cohesive forces between particles after lyophilization. Hence, it is envisaged that these formulations when administered will sink to the antrum of the stomach and be retained over a period for more of levodopa to be absorbed at its site of absorption.

\section{CONCLUSION}

Native locust bean gum is a poor directly compressible agent. However, it is usually used in tablet formulation by wet granulation method or polymer-polymer blending. Aqueous blending and lyophilization modified the gum, making it directly compressible. In addition, the matrices of the lyophilized polymer blends performed better than those of dry blended polymer with regards to hardness, resilience and density. It is envisaged that the matrices of the lyophilized polymer blends will exhibit better controlled release of levodopa than those of the dry polymer blends.

\section{REFERENCES}

1. Bernkop-Schnürch A. Chitosan and its derivatives: potential excipients for peroral peptide delivery systems. Int J Pharm 2000; 194: 1-13.

2. Chen L, Liu Z, Zhuo R. Synthesis and properties of degradable hydrogels of konjac glucomannan grafted acrylic acid for colon-specific drug delivery. Polymer 2005; 46: 6274-6281.

3. Chen $S$, Wu Y, Mi F, Lin $Y$, Yu L, Sung $H$. A novel $p H$ sensitive hydrogel composed of N,O-carboxymethyl chitosan and alginate cross-linked by genipin for protein drug delivery. J. Controlled Release 2004; 96 : 285-300.

4. LiU J, Lin S, Li L, Liu E. Release of theophylline from polymer blend hydrogels. Int. J. Pharm 2005; 298: 117-125.

5. Sogolova TI. Physical and physicochemical modification of polymers. Polymer Mechanics 1972; 8: 353-364.

6. Bhardwaj TR, Kanwar M, Lal R. Natural gums and modified natural gums as sustained release carriers. Drug Dev Ind Pharm 2000; 26: 1025-1038.

7. Billa $N$, Yuen $\mathrm{KH}$. Formulation variables affecting drug release from xanthan gum matrices at laboratory scale and pilot scale. AAPS Pharm Sci Tech 2000; 1: 1-8.

8. Ngwuluka N, Idiakhoa B, Nep E, Ogaji I, Okafor I. Formulation and evaluation of paracetamol tablets manufactured using the dried fruit of Phoenix dactylifera Linn as an excipient. Research in Pharmaceutical Biotechnology 2010; 2: 25-32.

9. Guo Y, Byrn SR, Zografi G. Effects of Lyophilization on the Physical Characteristics and Chemical Stability of Amorphous Quinapril Hydrochloride. Pharmaceutical Research 2000; 17: 930-936.

10. Vivoda $M, R o \AA_{j k a r} R, K$ metec $V$. The development of a quick method for amorphicity determination by isothermal microcalorimetry. Journal of Thermal Analysis and Calorimetry 2011; 105 (3): 1023-1030.

11. Tako M. Synergistic interaction between xanthan and tara-bean gum. Carbohydr. Polym 1991; 16: 239-252. 
12. Turquois T, Doublier J, Taravel F, Rochas $C$. Synergy of the $\kappa$-carrageenan-carob galactomannan blend inferred from rheological studies. Int. J. Biol. Macromol 1994; 16: 105-107.

13. Whitney SEC, Brigham JE, Darke AH, Reid JSG, Gidley MJ. Structural aspects of the interaction of mannanbased polysaccharides with bacterial cellulose. Carbohydr. Res 1998; 307: 299-309.

14. United States Pharmacopeia 32-NF27. General chapters: $<1174>$ Powder flow. The U.S. Pharmacopeial Convention Inc., Rockville, MD, USA 2009, pp. 688.

15. Jivraj M, Martini LG, Thomson CM. An overview of the different excipients useful for the direct compression of tablets. Pharm. Sci. Technol. Today 2000; 3: 5863.

16. Martinello T, Kaneko TM, Velasco MVR, Taqueda MES, Consiglieri VO. Optimization of poorly compactable drug tablets manufactured by direct compression using the mixture experimental design. Int. J. Pharm 2006; 322: 87-95.
17. Gupta S, Saini L. Effect of lyophilization and polymer compositions on solubility of aceclofenac solid dispersions. Journal of Advanced Pharmacy Education \& Research 2011; 2: 113-119.

18. Pillay V, Fassihi $R$. In vitro release modulation from crosslinked pellets for site-specific drug delivery to the gastrointestinal tract: II. Physicochemical characterization of calcium-alginate, calciumpectinate and calcium-alginate-pectinate pellets. J. Controlled Release 1999; 59: 243-256.

19. Picker-Freyer K, Dürig T. Physical mechanical and tablet formation properties of hydroxypropylcellulose: In pure form and in mixtures. AAPS PharmSciTech 2007; 8: 82-90.

20. Tsai $T$, San $Y$, Ho $H$, Wu J, Sheu M. Film-forming polymer-granulated excipients as the matrix materials for controlled release dosage forms. J. Controlled Release 1998; 51: 289-299. 